\title{
Full two-electron calculations of antiproton collisions with molecular hydrogen
}

\author{
Armin Lühr and Alejandro Saenz \\ Institut für Physik, AG Moderne Optik, Humboldt-Universität zu Berlin, Newtonstr. 15, D-12489 Berlin, Germany
}

(Received 23 October 2009; published 5 January 2010)

\begin{abstract}
Total cross sections for single ionization and excitation of molecular hydrogen by antiproton impact are presented over a wide range of impact energies from $1 \mathrm{keV}$ to $6.5 \mathrm{MeV}$. A nonperturbative time-dependent close-coupling method is applied to fully treat the correlated dynamics of the electrons. Good agreement is obtained between the present calculations and experimental measurements of single-ionization cross sections at high energies, whereas some discrepancies with the experiment are found around the maximum. The importance of the molecular geometry and a full two-electron description is demonstrated. The present findings provide benchmark results which might be useful for the development of molecular models.
\end{abstract}

DOI: 10.1103/PhysRevA.81.010701

PACS number(s): $34.50 . \mathrm{Gb}, 25.43 .+\mathrm{t}$

A central point of atomic and molecular physics is the description of charged particles moving in a Coulomb field. One of the simplest and most basic systems which provides an insight into dynamic processes of charged particles is the collision of antiprotons with atoms. The heavy mass of the antiproton allows, first, for a semiclassical theoretical approach and, second, for the investigation of "slow" ionizing collisions. In contrast to positively charged projectiles, for antiprotons there is no complication from charge transfer.

Further attention is drawn to this topic due to the upcoming Facility for Antiproton and Ion Research (FAIR) [1] with the international collaborations FLAIR (Facility for Low-energy Antiproton and Ion Research) [2] and SPARC (Stored Particle Atomic Research Collaboration) [3], both intending to investigate antiproton-driven processes and even kinematically complete collision experiments. However, the design of FLAIR already requires a reliable knowledge of low-energy antiproton cross sections of residual gases as, e.g., molecular hydrogen. These experimental efforts complement the recent intensive studies on antihydrogen at CERN aiming to test the CPT invariance and to disclose the nature of antimatter gravity.

Over the last two decades, remarkable progress in understanding the interactions between antiprotons and atoms has been achieved (see [4] and references therein). The theoretical description concentrates mainly on hydrogen and helium atoms. It was relatively easy to establish a full treatment of the former target, which only consists of one electron and one nucleus. For helium atoms, on the other hand, the dynamic electron-electron correlation effects turned out to be decisive, requiring much greater effort for their correct description. Due to the above-mentioned favorable properties, antiproton collisions on helium atoms became a benchmark system for studying electron correlation in atoms, stimulating a large number of calculations which employed various theoretical methods. During the last ten years, close-coupling calculations using either a spectral or spatial expansion of the two-electron wave function [5-8] yielded the most precise results, where the latter usually take advantage of large-scale computing facilities. They have provided cross sections for single and double ionization which are mostly in agreement with experiment for intermediate to high impact energies, while discrepancies still persist for low energies.
Antiproton collisions with molecules have been studied experimentally in a similar way as atoms concentrating mainly on ionization cross sections [9] and stopping powers [10] where for the latter rather diverse results were obtained at low energies. In contrast, the theoretical work on collisions involving molecules is still comparatively sparse (see [11] and references therein). Certainly, the description of a four-particle system like a hydrogen molecule, consisting of two electrons and two nuclei, is a further step in complexity compared to a helium atom. Recently, the ionization and excitation cross sections [11,12] as well as the stopping power [13] for antiproton impact on molecular hydrogen were calculated using spherical one-electron models for the hydrogen molecule [14]. They could mostly reproduce the experimental antiproton results for impact energies $E \geqslant 90 \mathrm{keV}$. The findings suggest, however, that for lower energies, molecular as well as electronelectron correlation effects are important and have to be considered. An earlier work by Ermolaev [15] turned out to be unsatisfactory for $E \leqslant 200 \mathrm{keV}$, reproducing atomic rather than molecular hydrogen. Furthermore, two calculations, both treating the target as a molecule, were performed by Sakimoto [16] and recently by the present authors [17] for molecular hydrogen ions. It was shown that the calculation of only three orientations of the molecular axis with respect to the projectile trajectory are sufficient to obtain the ionization cross section [17]. Currently new experimental data for antiproton collisions with molecular hydrogen are being produced using the Antiproton Decelerator (AD) facility at CERN [18].

In response to the renewed experimental activity and the limited theoretical understanding, a full two-electron closecoupling method for describing antiproton impacts on molecular targets has been developed. Converged cross sections for single ionization and excitation of molecular hydrogen are provided over a wide energy range from $1 \mathrm{keV}$ to $6.5 \mathrm{MeV}$ on a dense energy grid. They demonstrate the importance of a full two-electron description and of the molecular geometry including different orientations of the molecular axis as well as the differences between atomic and molecular targets.

The collision process is considered in a nonrelativistic semiclassical way using the impact parameter method (see [19]) which is known to be highly accurate for impact energies $E \gtrsim 1 \mathrm{keV}$ [6]. The quantum-mechanically treated electrons are exposed to the Coulomb potential of the molecular nuclei 
as well as the heavy projectile. The latter is assumed to move on a straight classical trajectory $\mathbf{R}(t)=\mathbf{b}+\mathbf{v} t$ given by the impact parameter $\mathbf{b}$ and its velocity $\mathbf{v}$, while $t$ is the time.

In the Born-Oppenheimer approximation, the total wave function of the hydrogen molecule separates into the product (atomic units are used unless stated otherwise)

$$
\tilde{\psi}_{k}^{(\Omega)}\left(\mathbf{r}_{1}, \mathbf{r}_{2}, \mathbf{R}_{\mathrm{nu}}\right)=\frac{\chi_{\nu j}^{(k)}\left(R_{\mathrm{nu}}\right)}{R_{\mathrm{nu}}} Y_{j}^{m}(\Theta, \Phi) \psi_{k}\left(\mathbf{r}_{1}, \mathbf{r}_{2} ; R_{\mathrm{nu}}\right),
$$

where $\chi_{\nu j}^{(k)}$ are the eigenfunctions of the molecular vibration, $Y_{j}^{m}$ are the spherical harmonics, and $\Omega \equiv(v, j, m)$ denotes the vibrational and rotational quantum numbers. $\mathbf{R}_{\mathrm{nu}}=\left(R_{\mathrm{nu}}, \Theta, \Phi\right)$ and $\mathbf{r}_{1,2}$ are the position vectors of the nuclei and the electrons, respectively. The wave function $\psi_{k}\left(\mathbf{r}_{1}, \mathbf{r}_{2} ; R_{\mathrm{nu}}\right)$ satisfies the electronic part of the timeindependent Schrödinger equation $\left(\hat{H}_{e}\right)$ for an unperturbed molecule at a fixed internuclear distance $R_{\text {nu }}$. The $\psi$ are obtained together with the eigenenergies $\epsilon$ in a full configurationinteraction (CI) calculation [20,21]. The two-electron configurations are constructed from correctly antisymmetrized products of orbitals which are eigenstates of the molecular hydrogen ion. The orbitals were obtained as in Ref. [17], where the radial part is expanded in $B$ splines and the angular part in spherical harmonics. More details on the extension of the close-coupling method from one-electron [17,22] to two-electron targets are provided in [23].

For a fixed $\mathbf{R}_{\text {nu }}$, the fully correlated wave function $\Psi$ of the two-electron target molecule interacting with the antiproton is obtained by the evolution of the time-dependent Schrödinger equation in real time, that is,

$$
i \frac{\partial}{\partial t} \Psi\left(\mathbf{r}_{1}, \mathbf{r}_{2}, t\right)=\left[\hat{H}_{e}+\hat{V}_{\mathrm{int}}\left(\mathbf{r}_{1}, \mathbf{r}_{2}, t\right)\right] \Psi\left(\mathbf{r}_{1}, \mathbf{r}_{2}, t\right),
$$

where the time-dependent interaction between the electrons and the projectile with charge $Z_{p}$ is expressed by

$$
\hat{V}_{\text {int }}\left(\mathbf{r}_{1}, \mathbf{r}_{2}, t\right)=-\frac{Z_{p}}{\left|\mathbf{r}_{1}-\mathbf{R}(t)\right|}-\frac{Z_{p}}{\left|\mathbf{r}_{2}-\mathbf{R}(t)\right|} .
$$

The interaction of the projectile with the nuclei, which leads only to an overall phase, is not considered here.

The time-dependent scattering wave function

$$
\Psi\left(\mathbf{r}_{1}, \mathbf{r}_{2}, t\right)=\sum_{k} c_{k}(t) \psi_{k}\left(\mathbf{r}_{1}, \mathbf{r}_{2}\right) e^{-i \epsilon_{k} t}
$$

is expanded in the normalized eigenstates $\psi_{k}$ of $\hat{H}_{e}$. Employing this expansion in Eq. (2) and projecting with $\psi_{k}$ leads to the usual set of coupled equations for every trajectory $\mathbf{R}(t)$,

$$
i \frac{\mathrm{d} c_{k}}{\mathrm{~d} t}=e^{i \epsilon_{k} t} \sum_{j} c_{j}\left\langle\psi_{k}\left|\hat{V}_{\mathrm{int}}\right| \psi_{j}\right\rangle e^{-i \epsilon_{j} t} .
$$

The two-electron interaction matrix elements in Eq. (5) canaccording to Eq. (3) - be expressed as a sum of one-electron matrix elements in the orbital basis. The full two-electron interaction matrix element between $\psi_{k}$ and $\psi_{j}$ is therefore the sum of the products between the CI coefficients of $\psi_{k}$ and $\psi_{j}$ and the one-electron matrix elements - calculated as in [17]between the orbitals of the corresponding CI configurations.
The coupled differential equations in Eq. (5) are integrated in a finite $z$ range of -50 a.u. $\leqslant z=v t \leqslant 50$ a.u. with the initial conditions $c_{k}\left[\mathbf{R}\left(t_{0}=-50 / v\right)\right]=\delta_{k 0}$, i.e., the target is initially in the electronic ground state $\psi_{0}$ with energy $\epsilon_{0}$. The probability for a transition into the final state $\psi_{k}$ at $t_{f}=50 / v$ for a fixed $\mathbf{R}_{\mathrm{nu}}$ is given by

$$
p_{k}\left(b, E ; R_{\mathrm{nu}}, \Theta, \Phi\right)=\left|c_{k}\left(b, v, t_{f} ; R_{\mathrm{nu}}, \Theta, \Phi\right)\right|^{2} .
$$

In accordance with [16], the transition probability

$$
\begin{aligned}
p_{k}(b, E)= & \int\left|\chi_{\nu j}\left(R_{\mathrm{nu}}\right) Y_{j}^{m}(\Theta, \Phi)\right|^{2} \\
& \times p_{k}\left(b, E ; R_{\mathrm{nu}}, \Theta, \Phi\right) \sin \Theta \mathrm{d} R_{\mathrm{nu}} \mathrm{d} \Theta \mathrm{d} \Phi
\end{aligned}
$$

becomes orientation-independent by integration over $\mathbf{R}_{\mathrm{nu}}$. The corresponding cross section

$$
\sigma_{k}(E)=2 \pi \int p_{k}(b, E) b \mathrm{~d} b
$$

can then be obtained by integration over $b$ as is done for atomic targets which are spherical symmetric. The total cross sections for ionization $\sigma_{\text {ion }}$ and bound-state excitation $\sigma_{\text {exc }}$ of the target can be obtained by summing up all partial cross sections $\sigma_{k}$ into states $k$ [as given in Eq. (8)] with $\epsilon_{k}>I_{\mathrm{H}_{2}}$ and $\epsilon_{0}<\epsilon_{k}<I_{\mathrm{H}_{2}}$, respectively, where $I_{\mathrm{H}_{2}}$ is the first ionization potential of the hydrogen molecule.

In this work, however, $p_{k}$ is approximated by

$$
p_{k}=\frac{1}{3}\left[p_{k}(0,0)+p_{k}\left(\frac{\pi}{2}, 0\right)+p_{k}\left(\frac{\pi}{2}, \frac{\pi}{2}\right)\right],
$$

using only the probabilities $p_{k}(\Theta, \Phi)$ for three orthogonal orientations of the internuclear axis with respect to the trajectory as is discussed in detail in Ref. [17]. It was shown that this approximation is equivalent to Eq. (7) if the integration is performed with a six-point quadrature formula [24]. Furthermore, excellent agreement between the results using Eqs. (7) and (9) were obtained for antiproton collisions with molecular hydrogen ions [16,17].

The dependence of the ionization cross section on the internuclear distance $R_{\text {nu }}$ in antiproton collisions with the molecular hydrogen ion and the hydrogen molecule was examined in [16] and [11], respectively. In both cases, an approximately linear dependence on $R_{\mathrm{nu}}$ around the expectation value $\left\langle R_{\mathrm{nu}}\right\rangle$ of the ground state was observed leading to Franck-Condon results, which were found to be very close to the exact cross sections obtained by an integration over $R_{\mathrm{nu}}$. In this work, the calculations are accordingly performed for $\left\langle R_{\mathrm{nu}}\right\rangle=1.4478$ a.u. The two-electron basis consists of 3080 singlet states with total azimuthal quantum numbers $M=0, \ldots, 4$ and a maximum energy of $\epsilon \approx 25$ a.u.

In Fig. 1, the results of the time-dependent close-coupling calculations for (a) single ionization and (b) bound-state excitation of the hydrogen molecule by antiproton impact are presented. The present results are compared with the data available in the literature. The single-ionization cross section in the top panel is in excellent agreement with the experimental measurements for energies above $85 \mathrm{keV}$ except for the data points at $500 \mathrm{keV}$. Below $85 \mathrm{keV}$, the experimental data show a small discontinuous step and increase to a higher maximum than the present results, with both maxima being situated around $40 \mathrm{keV}$. Note that in the extensive convergence studies 


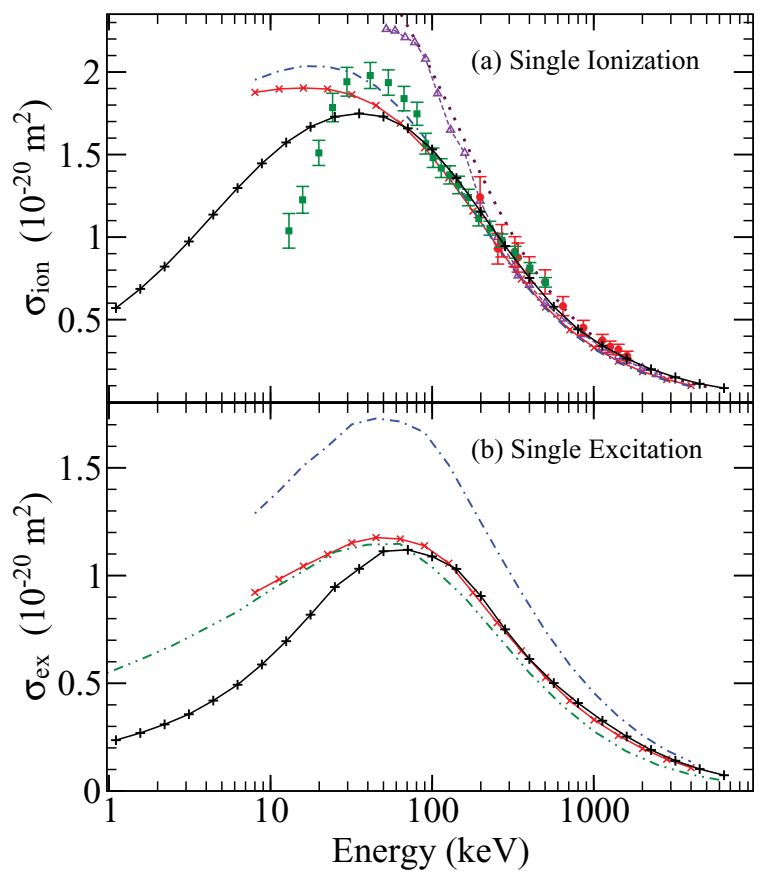

FIG. 1. (Color online) Cross sections for (a) single ionization $\sigma_{\text {ion }}$ and (b) excitation $\sigma_{\text {exc }}$ by antiproton impact. Black solid curve with pluses presents results for molecular hydrogen; red solid curve with $\mathrm{X}, \mathrm{H}_{2}$ model [11]; blue dashed-dotted curve, scaled hydrogen atom $\mathrm{H}_{\text {scal }}$ [11]; green double-dotted-dashed curve, hydrogen atom [22]; maroon dotted curve, two times hydrogen atom [11]; violet dashed curve with triangles, $\mathrm{H}_{\text {scal }}$ by Ermolaev [15]; green squares, CERN 94 [9]; red circles, CERN 90 [25].

performed in this work, an enlargement of the basis always led to smaller values of the maximum. Below the maximum, the experimental data fall off steeply in a similar way as the data for helium which were measured in the same occasion [9]. For helium, however, the two lowest energy data points were withdrawn after a recent remeasurement [4]. The currently produced experimental hydrogen molecule data for low impact energies may help to clarify the trend below the maximum.

The literature results obtained using a model potential and a hydrogen atom with scaled nuclear charge $Z=1.09$ $[11,12,14]$ are able to approximate the present calculations for energies above 50 and $100 \mathrm{keV}$, respectively. Though, they are throughout lower than the latter for these energies. For lower energies, the models yield evidently too large cross sections and in both cases show an atomic rather than a molecular slope by which they reveal their atomic nature. Below the maximum also the lack of electron-electron correlation effects can be expected to become severe as in the case of the helium atom [4,23]. The calculations by Ermolaev [15], using also a scaled hydrogen atom, are not satisfactory, since they follow for intermediate energies the data for a hydrogen atom multiplied by a factor of 2 .

The lower panel of Fig. 1 compares the present excitation cross sections for molecular hydrogen with the existing literature, i.e., the two already mentioned models $[11,14]$. Obviously, the scaled hydrogen atom is not capable of reproducing the excitation cross section for molecular hydrogen despite its reasonable results for ionization for $E>100 \mathrm{keV}$.

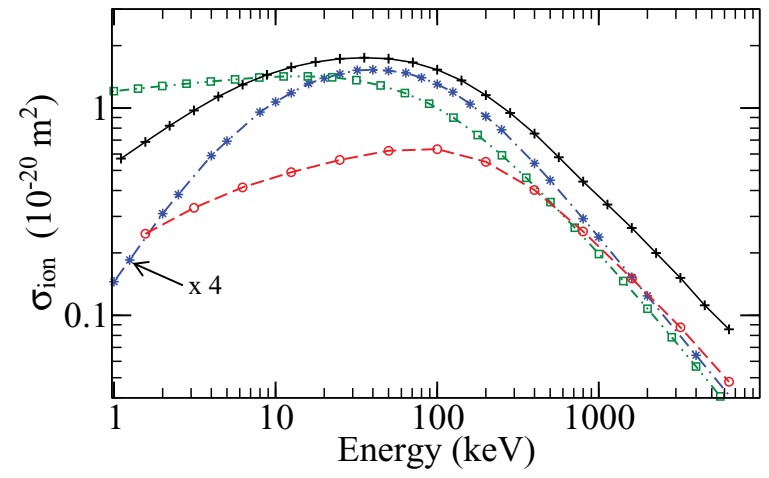

FIG. 2. (Color online) Comparison of single-ionization cross sections for antiproton impact on different targets. Black solid line with pluses presents results for molecular hydrogen; red dashed curve with circles, helium atom [23]; blue dot-dashed curve with stars, molecular hydrogen ion [17] (times 4); green double-dot-dashed curve with squares, hydrogen atom [22].

The model potential, on the other hand, is again an excellent approximation for energies above $50 \mathrm{keV}$. This might have been expected since the bound-state energies and oscillator strengths of the model potential were found to be in good agreement with those of the hydrogen molecule in contrast to the ones predicted by the scaled hydrogen atom [14]. Note that for energies above the maximum, the cross section for excitation of the hydrogen molecule is quite similar to that of atomic hydrogen, while for ionization it is rather comparable to twice the cross section of the hydrogen atom.

In Fig. 2, the single-ionization cross section of molecular hydrogen by antiproton collision is compared with results for the helium [23] and hydrogen atom [22] and the molecular hydrogen ion [17], where the latter is scaled by a factor of 4. The comparison shows that below the maximum, the curve for molecular hydrogen decreases much faster with decreasing energy than is the case for the hydrogen and helium atom. The molecular hydrogen curve is on the other hand qualitatively similar to that of the molecular ion. That is, especially for these energies, the qualitative shape of the ionization cross section seems to be different for atomic and molecular targets. At low energies, ionization occurs mainly in a small $b$ region close to the nuclei (cf. $b$-resolved transition probabilities, e.g., in $[22,23]$ ) where the electronic density and the expectation value of the electron velocity are high. In a close encounter of the antiproton on a molecular target, the electron cloud might be more efficiently moved away from the projectile toward the other nuclei, especially for the molecular ion, since there is in contrast to atoms always one positive particle which is not neutralized by the antiproton.

At high energies, the single-ionization cross sections for helium and for four times the molecular hydrogen ion are both similar to the curve of the hydrogen atom, while the results for molecular hydrogen are in good agreement with twice the curve for the hydrogen atom [cf. Fig. 1(a)]. For these energies, distant encounters are dominating the ionization process. Accordingly, details of the targets such as the exact distribution of the positive charges become less important, and the cross sections are mostly determined by the ionization potential. 


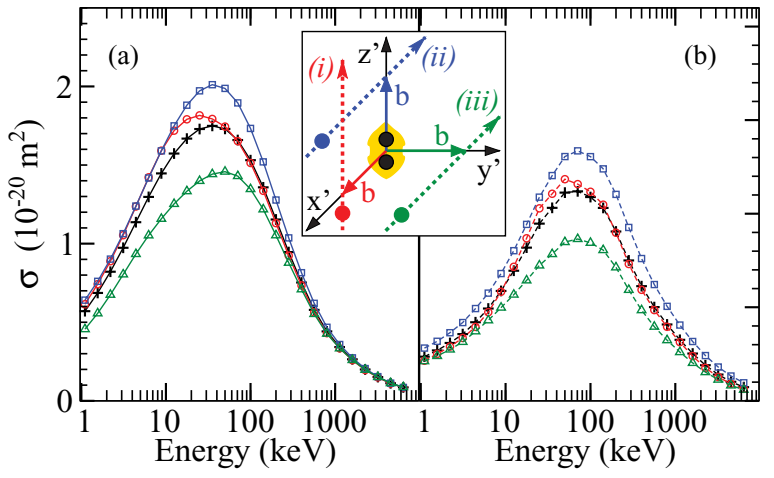

FIG. 3. (Color online) Cross sections for (a) single ionization and (b) excitation of molecular hydrogen by antiproton collisions for different molecular orientations. Black pluses, orientationally averaged; red circles, (i); blue squares, (ii); green triangles, (iii). The inset shows a sketch of the three orientations in the molecule-fixed frame.

In Fig. 3, the dependence on the orientation of the molecular axis with respect to the antiproton trajectory is presented. The cross sections $\sigma(\Theta, \Phi)$ for (a) ionization and (b) excitation are given for the three orthogonal orientations $(\Theta, \Phi)=(\mathrm{i})(0,0)$, (ii) $(\pi / 2,0)$, and (iii) $(\pi / 2, \pi / 2)$ (see the sketch in Fig. 3 and Ref. [17]) revealing the following results. First, the curves for the three different orientations differ in general and especially around the maximum where also the average in Fig. 1(a) differs most from the experimental data. Here, additional orientations might improve the average curve. Second, the calculation of only the parallel orientation (i) reproduces for energies above the maximum the orientationally averaged results with less than $3 \%$ relative deviation. Third, for lower energies at which close collisions become dominant, the consideration of the molecular geometry is inevitable. In contrast to the findings for the molecular hydrogen ion [17], the curves of orientation (i) are close to those of (ii) for ionization below the maximum and of (iii) for excitation below $5 \mathrm{keV}$. In general, the differences among the cross sections for the three orientations are less pronounced than for the molecular hydrogen ion. This might be due to the smaller internuclear distance and the two electrons of the hydrogen molecule making it a more spherical target.

In conclusion, theoretical data are presented for single ionization and excitation of molecular hydrogen by antiproton impact for a wide energy range obtained with a two-electron time-dependent close-coupling method. The experimental data are in good agreement with the present calculations at high energies but are larger around the maximum. For energies below the maximum, the ionization cross section decreases with decreasing energy much faster than in the cases for the hydrogen and helium atom but in a similar way as for the molecular hydrogen ion, revealing the differences between atoms and molecules. Furthermore, the importance of the molecular geometry and a full two-electron description is demonstrated. The present work should motivate new experimental efforts for molecular targets at low impact energies to confirm and further extend the gained insight. Additionally, it provides benchmark data for molecular collisions in general and for single ionization and excitation of molecular hydrogen by antiproton impact, in particular, which might be useful for the development of molecular models.

The method should be further exploited to extract also quantities like, e.g., electron-energy spectra or differential excitation cross sections. Such results may allow, especially at low energies, the elimination of the diversity of results for the stopping power obtained in different experiments $[10,13]$.

The authors acknowledge stimulating correspondence with H. Knudsen. This work was supported by BMBF (FLAIR Horizon) and Stifterverband für die deutsche Wissenschaft.
[1] http://www.gsi.de/fair/

[2] http://www.oeaw.ac.at/smi/flair/

[3] http://www.gsi.de/fair/experiments/sparc/

[4] H. Knudsen et al., Phys. Rev. Lett. 101, 043201 (2008).

[5] T. G. Lee, H. C. Tseng, and C. D. Lin, Phys. Rev. A 61, 062713 (2000).

[6] A. Igarashi et al., Nucl. Instrum. Methods Phys. Res. B 214, 135 (2004).

[7] D. R. Schultz and P. S. Krstić, Phys. Rev. A 67, 022712 (2003).

[8] M. Foster, J. Colgan, and M. S. Pindzola, Phys. Rev. Lett. 100, 033201 (2008).

[9] P. Hvelplund et al., J. Phys. B 27, 925 (1994).

[10] E. Lodi Rizzini et al., Phys. Rev. Lett. 89, 183201 (2002).

[11] A. Lühr and A. Saenz, Phys. Rev. A 78, 032708 (2008).

[12] A. Lühr and A. Saenz, Hyperfine Interact. 194, 59 (2009).

[13] A. Lühr and A. Saenz, Phys. Rev. A 79, 042901 (2009).
[14] A. Lühr, Y. V. Vanne, and A. Saenz, Phys. Rev. A 78, 042510 (2008).

[15] A. M. Ermolaev, Hyperfine Interact. 76, 335 (1993).

[16] K. Sakimoto, Phys. Rev. A 71, 062704 (2005).

[17] A. Lühr and A. Saenz, Phys. Rev. A 80, 022705 (2009).

[18] H. Knudsen (private communication).

[19] B. H. Bransden and M. R. C. McDowell, Charge Exchange and the Theory of Ion-Atom Collisions (Clarendon, Oxford, 1992).

[20] A. Apalategui, A. Saenz, and P. Lambropoulos, Phys. Rev. Lett. 86, 5454 (2001).

[21] A. Apalategui and A. Saenz, J. Phys. B 35, 1909 (2002).

[22] A. Lühr and A. Saenz, Phys. Rev. A 77, 052713 (2008).

[23] A. Lühr and A. Saenz (unpublished).

[24] L. F. Errea et al., J. Phys. B 30, 3855 (1997).

[25] L. H. Andersen et al., J. Phys. B 23, L395 (1990). 